\title{
Mechanistic insights into acyclovir-polyethylene glycol 20000 binary dispersions
}

\author{
Krishnamoorthy Venkateskumar, Subramani Parasuraman ${ }^{1}$, Raju Gunasunderi², Krishnan Sureshkumar ${ }^{3}$, \\ M. Muralidhar Nayak ${ }^{4}$, Syed Adnan Ali Shah ${ }^{5}$, Khoo Kassen, Heng Wei Kai \\ Units of Pharmaceutical Technology and ${ }^{~}$ Pharmacology, Faculty of Pharmacy, AIMST University, Semeling 08100, ${ }^{2}$ Colloid Interface Science \\ Centre, Centre of Excellence, Malaysian Rubber Board, Experiment Station, Sungai Buloh, Selangor DE, Malaysia, ${ }^{3} \mathrm{KMCH}$ College of \\ Pharmacy, Coimbatore, Tamil Nadu, ${ }^{4}$ Spectroscopy Analytical Test Facility, Indian Institute of Science, Bengaluru, Karnataka, India, ${ }^{5}$ Faculty \\ of Pharmacy, Universiti Teknologi Mara, Puncak Alam Campus, Selangor, Dahrul Ehsan, Malaysia
}

\begin{abstract}
Objective: The objective of this study is to provide a mechanistic insight into solubility enhancement and dissolution of acyclovir (ACY) by polyethylene glycol20000 (PEG20000). Materials and Methods: Solid dispersions with differing ratios of drug (ACY) and carrier (PEG20000) were prepared and evaluated by phase solubility, in vitro release studies, kinetic analysis, in situ perfusion, and in vitro permeation studies. Solid state characterization was also done by Powder X-Ray Diffraction (PXRD), Differential Scanning Calorimetry (DSC), Fourier Transform Infrared spectroscopy (FT-IR) analysis and surface morphology was assessed by Polarizing Microscopic Image (PMI) analysis, Scanning Electron Microscopy (SEM), Atomic Force Microscopy (AFM), and Nuclear Magnetic Resonance (NMR) analysis. Results: Thermodynamic parameters proved the solubilization effect of carrier. The aqueous solubility and dissolution of ACY were increased in all samples. Formation of solid solution, crystallinity reduction, and absence of interaction between drug and carrier was proved by XRD, DSC, and FTIR analysis. The particle size reduction and change in surface morphology were confirmed by SEM and AFM and analysis. The permeation coefficient and amount of drug diffused was higher in samples as compared to ACY. The stability was high in dispersions, and it was proved by NMR analysis. Conclusion: The mechanical insights into the enhancement of solubility and dissolution could be used as a platform to improve the aqueous solubility for other poor water soluble drugs.
\end{abstract}

Key words: Acyclovir, crystallinity, drug-polymer miscibility, solid dispersions, solid solution

\section{INTRODUCTION}

The poor aqueous solubility of chemical entities is one of the major challenges in pharmaceutical development. ${ }^{[1]}$ Several strategies such as salt formation, complexation, micelle formation, drug derivatization, solid state manipulation, inclusion of surfactants, micronization or nanonization, spray drying of solid lipid nanoparticles, and usage of solid dispersions (SDs) have been adopted for enhancing drugs' dissolution. SDs is considered to be one of the most successful strategies to improve

\section{Address for correspondence:}

Dr. Krishnamoorthy Venkateskumar,

Unit of Pharmaceutical Technology, Faculty of Pharmacy, AIMST

University, Semeling 08100 Bedong, Kedah, Darul Aman, Malaysia.

E-mail: venkateskumar@aimst.edu.my

\begin{tabular}{|l|l|}
\hline \multicolumn{2}{|c|}{ Access this article online } \\
\hline Quick Response Code: & Website: \\
\hline & www.jpionline.org \\
\cline { 2 - 3 } & DOI: \\
\hline
\end{tabular}

aqueous solubility and dissolution profile. Numerous studies have focused on SDs and their advantages. ${ }^{[2]}$ Through this study, we attempted to provide mechanical insights into the process of drug release from a model SD involving an antiviral drug and a hydrophilic carrier, and the aspects that contribute to the stability of resulting SDs.

Acyclovir (ACY) [9-(2-hydroxyethoxylmethyl) guanine], a synthetic purine nucleoside and its absorption in gastrointestinal tract (GIT) is slow, variable, and incomplete. The bioavailability of ACY after oral administration ranges from $10 \%$ to $30 \%{ }^{[3]}$ These parameters made ACY as the ideal choice for this study.

This is an open access article distributed under the terms of the Creative Commons Attribution-NonCommercial-ShareAlike 3.0 License, which allows others to remix, tweak, and build upon the work non-commercially, as long as the author is credited and the new creations are licensed under the identical terms.

For reprints contact: reprints@medknow.com

How to cite this article: Venkateskumar $\mathrm{K}$, Parasuraman $\mathrm{S}$, Gunasunderi R, Sureshkumar K, Nayak MM, Shah SA, et al. Mechanistic insights into acyclovir-polyethylene glycol 20000 binary dispersions. Int J Pharma Investig 2016;6:194-200. 
Many crystalline and amorphous carriers have been used to formulate SDs, and their success has been well-reported. Various grades of polyethylene glycol (PEG) have been tried for solubility enhancement, and the effect of the molecular weight of PEG on drug release has also been reported in similar studies. ${ }^{[4]}$ The effect of PEG20000 on the solubility and drug release of ACY from the resulting SDs were evaluated using a wide spectrum of advanced characterization techniques.

\section{MATERIALS AND METHODS}

\section{Materials}

ACY was obtained as a gift sample from CCM Duo Pharma Sdn BhD, Klang, Malaysia. PEG (PEG20000) was purchased from Sigma-Aldrich (Sigma-Aldrich Chemie, Steinheim Germany) and used to form the matrix. All other materials used were of analytical grade.

\section{Phase solubility studies}

ACY and PEG20000 at specific proportions (1:5, 1:20, and 5:1) were added to $25 \mathrm{~mL}$ distilled water in separate screw-capped bottles, agitated in an orbital shaker (Remi Ltd., Mumbai, Maharashtra, India), and incubated separately at $25^{\circ} \mathrm{C}$ and $37^{\circ} \mathrm{C}$ for $24 \mathrm{~h}$. Samples containing pure ACY and water were used as control. After $24 \mathrm{~h}$, the solutions were filtered, suitably diluted and analyzed spectrophotometrically at $253 \mathrm{~nm}$ (UV-1700, UV-Vis Spectrophotometer, Shimadzu, Japan). Thermodynamic parameters such as enthalpy $(\Delta \mathrm{H})$, entropy $(\Delta \mathrm{S})$, and Gibbs free energy $(\Delta G)$ were calculated. ${ }^{[5]}$

\section{Preparation of solid dispersions}

SDs with differing drug-polymer ratios (1:5, 1:20, and 5:1) were prepared by solvent method using $0.1 \mathrm{~N} \mathrm{HCl}$ as a solvent. The dispersions contain $20 \%, 5 \%$ and $80 \%$ W/W of ACY, respectively and the samples are coded as: APEG20 15, APEG20 120 and APEG20 51. ACY and PEG20000 were weighed as per drug: carrier ratios and dissolved in $0.1 \mathrm{~N} \mathrm{HCl}$ to form a clear solution. The solution was slowly heated on a water bath with stirring until it formed a viscous solid mass. The contents were then mixed well till it solidified. The mass was scrapped, powdered well in a mortar and stored in a desiccator until further studies. ${ }^{[4-7]}$ Drug content, dissolution studies, kinetic analysis, and solid-state characterization of the SDs were carried out. Surface morphology was done by scanning electron microscopy (SEM) and atomic force microscopy (AFM) analysis.

\section{Drug content}

A $25 \mathrm{mg}$ of SDs were dissolved in $25 \mathrm{~mL}$ of analytical media and filtered using a Whatman filter paper. The filtrate was diluted and measured in a UV-Vis spectrophotometer (UV-1700, Shimadzu, Japan) at $253 \mathrm{~nm} \cdot{ }^{[4-7]}$

\section{Dissolution studies}

An amount of SDs equivalent to $25 \mathrm{mg}$ of ACY were added to $900 \mathrm{~mL}$ of $0.1 \mathrm{~N} \mathrm{HCl}$, in a dissolution apparatus (Electro Labs,
Mumbai, India) and studies were conducted at $50 \mathrm{rpm}$ and a temperature of $37 \pm 0.5^{\circ} \mathrm{C}$. At periodic time intervals, $5 \mathrm{~mL}$ of sample was withdrawn and replenished with fresh dissolution medium to maintain sink conditions. The withdrawn samples were suitably diluted, and the amount of ACY present was measured in a UV-Vis spectrophotometer at $253 \mathrm{~nm} .{ }^{[6,7]}$ Various dissolution parameters, such as percentage of drug released at different time intervals (\%DP), percentage dissolution efficiency $(\% \mathrm{DE})$, relative dissolution rate, area under the curve, mean dissolution time, mean residence time (MRT), variance dissolution time, dissolution half-life $\left(\mathrm{t}_{50 \%}\right)$, and time taken to release $85 \%$ of drug $\left(\mathrm{t}_{85 \%}\right)$, were calculated by using DD solver program in MS Excel 2007 (Microsoft Inc, USA). ${ }^{[8]}$

\section{Kinetic analysis}

The drug release data were mapped with various release kinetic models (first-order, Higuchi, Hixson-Crowell cube root, Korsemeyer-Peppas and zero-order model), to ascertain the possible kinetics of drug release from SDs. ${ }^{[6-8]}$

\section{Solid state characterization}

Powder X-ray diffraction (PXRD), differential scanning calorimetry (DSC), Fourier transform infra-red spectroscopy (FT-IR), polarizing microscopic image (PMI) analysis, and nuclear magnetic resonance (NMR) analysis were carried out for solid state characterization of samples. Surface morphology of the SDs was assessed by SEM and AFM analysis. ${ }^{[9-12]}$

Powder X-ray diffraction patterns were recorded on a powder X-ray diffractometer (Philips PW1729, Eindhoven, Netherlands) using $\mathrm{Ni}$-filtered $\mathrm{Cu} \mathrm{K} \alpha$ radiation at a voltage of $35 \mathrm{kV}$ and $20 \mathrm{~mA} \cdot{ }^{[9-12]}$

DSC curves were obtained in differential scanning calorimeter (DSC-821, Mettler-Toledo International Inc. Columbus, USA). Samples weighing 5-10 mg were placed in sealed aluminum DSC pans with a pinhole to prevent pressure buildup and heated in a flowing atmosphere of nitrogen $(20 \mathrm{ml} / \mathrm{min}$.). Each sample was heated from $10^{\circ} \mathrm{C}$ to $300^{\circ} \mathrm{C}$ at the rate of $10^{\circ} \mathrm{C} / \mathrm{min} \cdot{ }^{[9-12]}$

FT-IR spectra of pure ACY and the formulated SDs were obtained on a FT-IR spectrometer (Perkin-Elmer 1600, PerkinElmer Inc., USA) equipped with a DTSG detector by conventional $\mathrm{KBr}$ pellet method. ${ }^{[9-12]}$ The scan range was $4000-500 / \mathrm{cm}$ with a resolution of $4 / \mathrm{cm}$.

PMI analysis was carried out on a polarizing optical microscope (BX51P Olympus, Olympus Corporation, Japan) equipped with $100 \mathrm{~W}$ halogen lamp housing, an ND25 neutral density filter, and a ProgRes C3 CCD camera was employed. The temperature range at the adjustable hot stage was $-80^{\circ} \mathrm{C}$ to $6000^{\circ} \mathrm{C}$. ProgRes capture Pro 2.5 software (JENOPTIK Laser, Optik, Systeme GmbH, Goeschwitzer Strasse, Germany) was used to analyze the images, and Linksys 32 was used to control the hot-stage temperature modulator. ${ }^{[9-12]}$ 
SEM analysis was carried out in a scanning electron microscope (JEOL JMS-840A- JEOL USA, Inc., MA, USA). The accelerating voltage was $10 \mathrm{kV}$ at a working distance of $\sim 10 \mathrm{~mm}$. Samples were gold coated before imaging. ${ }^{[9-12]}$

AFM images of the samples were recorded on a MultiMode AFM Dimension Icon 3000 equipment (Bruker corporation, Tokyo) equipped with humidity control (Triton Technology, UK) and images were taken at room temperature in a tapping mode with a NanoScope IIIa controller (Digital Instruments, NJ, USA). The images were obtained at a scan rate of $1.50 \mathrm{~Hz}$ and scan point of $512 \mathrm{~nm}$. All data were batch processed using Scanning Probe Image Processor, SPIP 5.1.1, (Image Metrology A/S, Horsholm, Denmark). ${ }^{[9-12]}$

NMR analysis was carried out using a NMR spectrometer (Bruker 300, Bruker Corporation, Tokyo) operated at $300 \mathrm{MHz}$. Data processing was carried out using sine bell software. ${ }^{[9-12]}$

\section{In vitro intestinal permeation studies In situ intestinal absorption study}

Healthy, adult, male Sprague-Dawley rats, weighing $180 \pm 20 \mathrm{~g}$ were used for the study. The study was conducted according to the guidelines of the Animal Research Review Panel. The study was conducted with prior approval from AIMST University's Human and Animal Ethics Committee (AUHAEC19/FOP/SP/2014).

The animals were anesthetized with intraperitoneal injection of pentobarbital $(60 \mathrm{mg} / \mathrm{kg})$, and they were placed on a heated pad to maintain a normal body temperature. The small intestine of the rats was surgically exposed, and $10 \mathrm{~cm}$ of jejunum was ligated for perfusion and cannulated and connected with the perfusion assembly. Blank perfusion buffer was infused for 10 min using a syringe pump followed by perfusion of SDs at a flow rate of $0.2 \mathrm{~mL} / \mathrm{min}$ for $60 \mathrm{~min}$. At periodic time intervals (every $10 \mathrm{~min}$ ), the perfusate was collected, and ACY content was measured using UV-Vis spectrophotometer at $253 \mathrm{~nm}$ and the effective permeability coefficient $\left(P_{\text {eff }}\right)$ was calculated. ${ }^{[13,14]}$

\section{In vitro intestinal permeation studies}

Male Sprague-Dawley rats $(180 \pm 20 \mathrm{~g})$ were sacrificed by cervical dislocation, and duodenal part of the small intestine was isolated. The segment was thoroughly washed with cold Ringer's solution. SDs equivalent to $10 \mathrm{mg}$ of ACY were dispersed in phosphate buffer ( $\mathrm{pH} 7.4$ ) and injected into the duodenum using a syringe, and both the ends of the intestine were tightly ligated. The segment was placed in a compartment containing $30 \mathrm{~mL}$ of phosphate-buffered saline ( $\mathrm{pH} \mathrm{7.4)}$ and maintained at a temperature of $37^{\circ} \mathrm{C}$ with continuous aeration. At periodic time intervals, the sample was taken and suitably diluted; its absorbance was measured at $253 \mathrm{~nm}$ using a UV-Vis spectrophotometer. The percentage of drug diffused across the intestinal segment was calculated. ${ }^{[13,14]}$

\section{Stability studies}

The prepared SDs were stored in closed glass vials under a controlled environment $(75 \%$ relative humidity $[\mathrm{RH}]$ and a temperature of $45^{\circ} \mathrm{C}$ ) in a stability chamber (Thermo Lab, India) for 6 months. Samples were removed after 3 and 6 months and evaluated for drug content and release profiles. ${ }^{[6-8]}$

\section{Statistical analysis}

The values were presented as a mean of three values. The statistical significance of the formulations was compared with that of control using analysis of variance followed by Bonferroni post hoc test. Statistical analysis was performed using Instat prism version 3.06 (Graph pad Inc., USA). A $P<0.05$ was considered as statistical significance.

\section{RESULTS}

The phase solubility data showed a linear increase in drug solubility with an increase in carrier content and temperature. The Gibbs free energy $(\Delta G)$ values were negative at all concentrations of carrier [Table 1]. The drug content in all SDs was in the range of $97-99 \%$.

In vitro release profiles of the SDs are shown in Figure 1a. The percentage of cumulative release of pure ACY was found to be $40 \%$ in $1 \mathrm{~h}$, whereas the SDs showed a significant $(P<0.001)$ increase in release rate during the same period (about $90 \%$ for SDs with drug: carrier ratio of 1:20). The drug release from SDs increased with increase in carrier content. The dissolution parameters such as percentage drug released (\%DP) were found to be increased whereas parameters such as MRT, half-life $\left(\mathrm{t}_{50 \%}\right)$, and $\mathrm{t}_{85 \%}$ were found to be decreased with increase in carrier content [Table 2]. The $r^{2}$ values of the Korsmeyer-Peppas model [Table 3] in all SDs were within the range of $0.943-0.993$ suggesting it as the best fitting model to describe the drug release from SDs.

Solid state characterization of SDs was studied using various characterization techniques and the results are provided as data and in images as PXRD [Figure 2a], DSC [Figure 2b], FTIR [Figure 2c], PMI [Figure 3a-c], SEM [Figure 4a-f], AFM [Figure 5a-c] and NMR analysis [Table 4]. The findings of the solid state characterizations suggest that crystallinity of ACY had reduced, formation of solid solution, alteration of structural morphology of ACY in the formulated SDs in comparison with pure ACY.

The enhanced $P_{\text {eff }}$ values, enhancement ratio and percentage of drug diffused from in situ perfusion and intestinal permeation studies of optimized SDs revealed their increased absorption potential in comparison to pure ACY [Table 5]. These findings clearly prove the potential of optimized SDs in improving oral delivery.

The release profiles of the samples after the stability studies are shown in Figure $1 \mathrm{~b}$ and 1c, respectively. It was also observed that there was not much significant change in the SDs during the study, suggesting that the samples were stable at the tested conditions. 
Table 1: Thermodynamic parameters of acyclovir physical mixtures with polyethylene glycol 20000

\begin{tabular}{lcccccc}
\hline Temperature $\left({ }^{\circ} \mathbf{C}\right)$ & Slope & Intercept & $\mathbf{K a ~}\left(\mathbf{m}^{-1}\right)$ & $\Delta \mathbf{G ~ ( k J / m o l )}$ & $\Delta \mathbf{H ~ ( k J / m o l )}$ & $\Delta \mathbf{S}(\mathbf{J} / \mathbf{m o l ~ K})$ \\
\hline 25 & 40.788 & 24.236 & -0.042 & -2.375 & -2.375 & -2.367 \\
37 & 156.341 & 22.602 & -0.045 & -2.465 & -2.465 & -2.457 \\
\hline
\end{tabular}

Ka: Stability constant, $\Delta \mathrm{G}:$ Gibbs free energy, $\Delta \mathrm{H}$ : Enthalpy and $\Delta \mathrm{S}$ : Entropy

Table 2: Dissolution parameters of acyclovir - polyethylene glycol 20000 dispersions

\begin{tabular}{lccccccccccc}
\hline Code & DP05 & DP30 & DP60 & \% DE & RDR & AUC & MDT & MRT & VDT & $\mathbf{t}_{50 \%}$ (min) & $\mathbf{t}_{85 \%}$ (min) \\
\hline ACY & $6.62(2.17)$ & $15.44(1.48)$ & $38.48(3.95)$ & 0.178 & 0.373 & 1068.97 & 32.24 & 27.94 & 388.0 & $>60$ & $>60$ \\
APEG20 15 & $7.68(3.17)$ & $75.70(0.64)$ & $92.28(2.64)$ & 0.602 & 0.566 & 3851.83 & 18.26 & 17.30 & 189.0 & 15 & 43 \\
APEG20 120 & $8.63(0.84)$ & $74.70(1.02)$ & $97.54(3.12)$ & 0.614 & 0.560 & 3896.11 & 19.00 & 15.39 & 165.5 & 22 & 32 \\
APEG20 51 & $7.22(1.14)$ & $27,344(1.26)$ & $47.29(2.46)$ & 0.214 & 0.501 & 1535.72 & 27.52 & 29.96 & 380.0 & $>60$ & $>60$
\end{tabular}

A value in parenthesis indicates standard deviation, DP05, DP30 and DP6o: Percentage drug released at $5 \mathrm{~min}, 30 \mathrm{~min}$ and $60 \mathrm{~min}, \%$ DE: Percentage dissolution efficiency, RDR: Relative dissolution rate at specific time intervals, AUC: Area under the curve, MDT: Mean dissolution time, MRT: Mean residence time, VDT: Variance dissolution time, $\mathrm{t}_{50 \%}$ : Dissolution half-life, $\mathrm{t}_{85 \%}$ : Time taken to release $85 \%$ of drug, ACY: Acyclovir

Table 3: Release kinetic parameters of acyclovir - polyethylene glycol 20000 solid dispersions

\begin{tabular}{|c|c|c|c|c|c|c|c|c|c|c|c|}
\hline \multirow[t]{2}{*}{ Code } & \multicolumn{2}{|c|}{ Zero order } & \multicolumn{3}{|c|}{ First order } & \multicolumn{2}{|c|}{ Higuchi } & \multicolumn{2}{|c|}{ Hixson Crowell } & \multicolumn{2}{|c|}{ K-P } \\
\hline & $r^{2}$ & $\mathrm{~K}_{0}$ & $r^{2}$ & Slope & $K_{1}$ & $r^{2}$ & Slope & $r^{2}$ & Slope & $r^{2}$ & $n$ \\
\hline $\mathrm{ACY}$ & 0.796 & 0.998 & 0.110 & 0.009 & 0.020 & 0.857 & 8.24 & 0.751 & 0.012 & 0.993 & 0.257 \\
\hline APEG20 15 & 0.808 & 1.880 & 0.978 & 0.002 & 0.004 & 0.933 & 12.47 & 0.977 & 0.012 & 0.943 & 0.579 \\
\hline APEG20 120 & 0.898 & 1.912 & 0.936 & 0.018 & 0.041 & 0.882 & 12.41 & 0.961 & 0.011 & 0.952 & 0.782 \\
\hline APEG20 51 & 0.939 & 0.804 & 0.964 & 0.004 & 0.010 & 0.935 & 5.234 & 0.958 & 0.003 & 0.977 & 0.737 \\
\hline
\end{tabular}

$\mathrm{K}_{0}$ : Zero order release constant, $\mathrm{K}_{\mathrm{i}}$ : First order release rate constant, $n$ release exponent, K-P: Korsmeyer-Peppas model

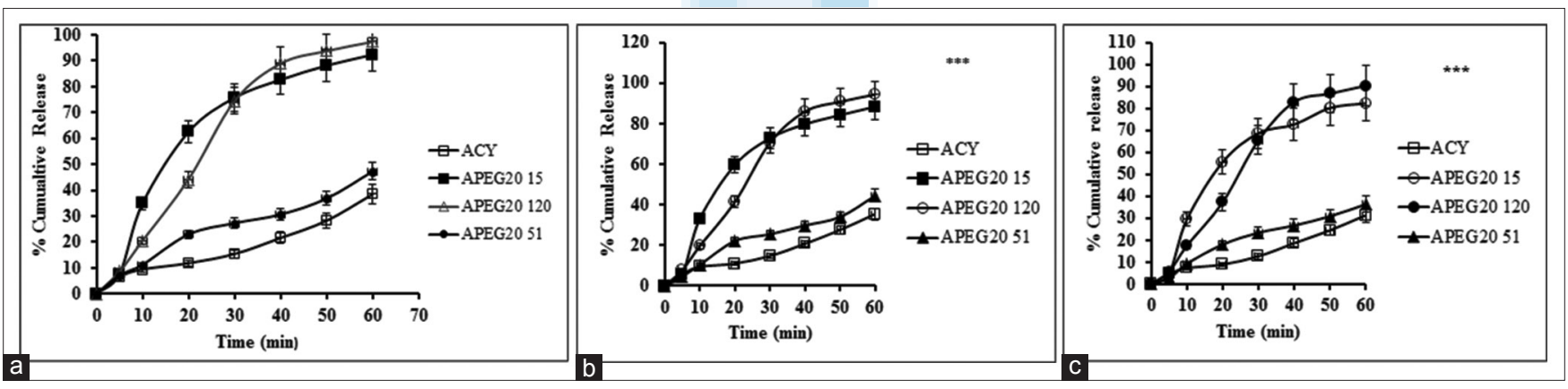

Figure 1: In vitro release profiles of acyclovir and solid dispersions (SDs) (a) APEG20 120 showed significant $\left({ }^{* * *} P<0.001\right)$ increase in release rate compared with that of pure acyclovir, APEG20 15 and APEG20 51. ${ }^{* \star *} P<0.001$ compared with that of pure acyclovir, APEG20 15 and APEG20 51 (one-way analysis of variance followed by Bonferroni post hoc test); (b) in vitro release profile of acyclovir and solid dispersions (mean \pm standard deviation; $n=3$ ) after 3 months; and (c) in vitro release profile of acyclovir and solid dispersions (mean \pm standard deviation; $n=3$ ) after 6 months. ACY: Acyclovir

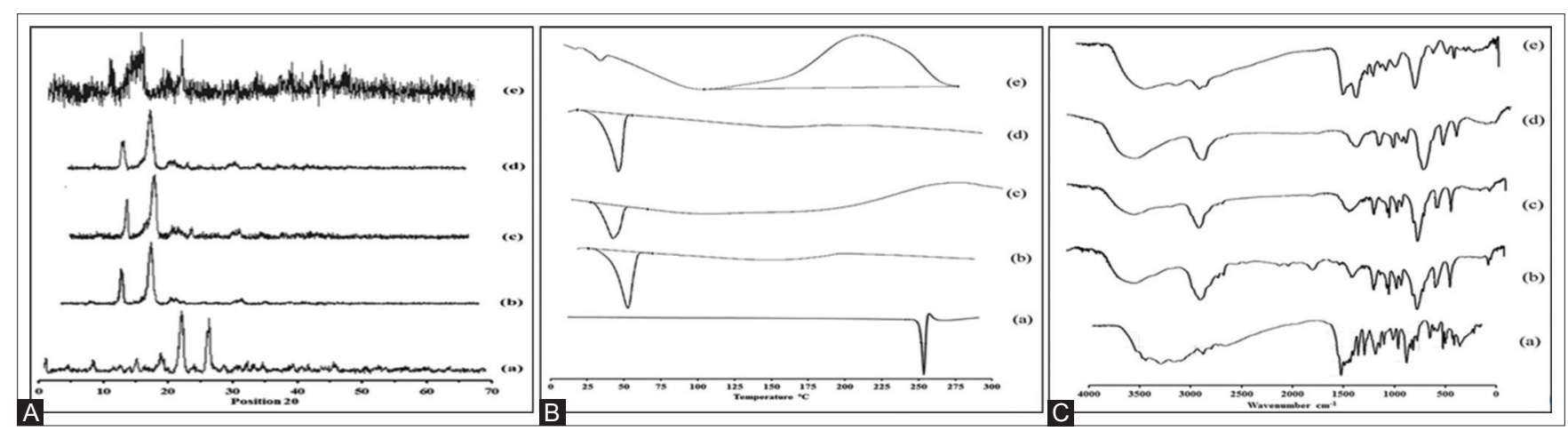

Figure 2: (A) Powder X-ray diffraction patterns (a) acyclovir (b) polyethylene glycol 20000 (c) APEG20 15 (d) APEG20 120 and (e) APEG20 51 ; (B) DSC thermograms (a) acyclovir (b) PEG20000 (c) APEG20 15 (d) APEG20 120 and (e) APEG20 51; (C) FT-IR spectra (a) acyclovir (b) polyethylene glycol 20000 (c) APEG20 15 (d) APEG20 120 and (e) APEG20 51 


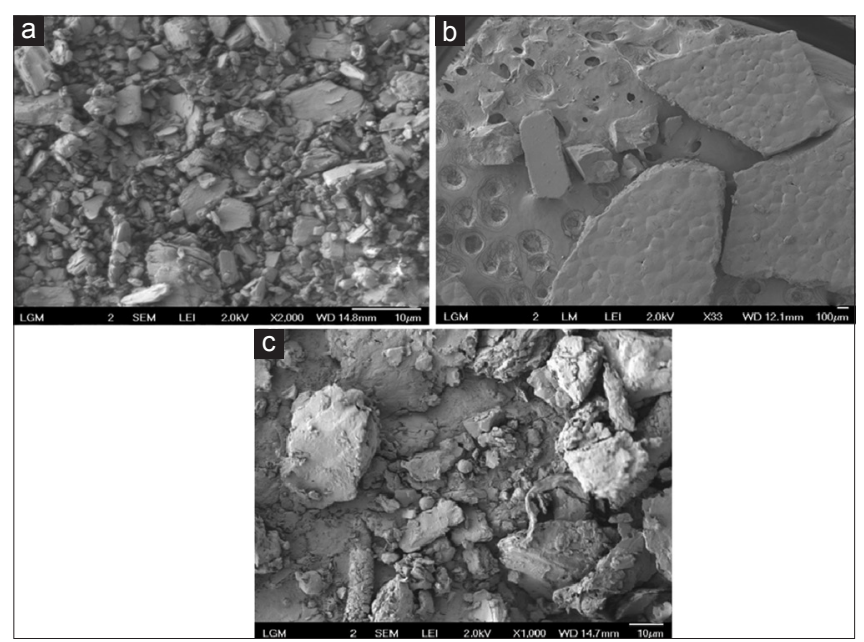

Figure 3: Scanning electron microscope images of (a) acyclovir (x2000), (b) polyethylene glycol 20000 (x33) and (c) APEG 120 (x1000)

\section{DISCUSSION}

The phase solubility study findings indicate the formation of weak water-soluble complexes between drug and the carrier. The negative thermodynamic parameters $(\Delta \mathrm{G}, \Delta \mathrm{H}$ and $\Delta \mathrm{S})$ clearly demonstrate the spontaneity of a drug's solubilization process and solubilization effect of the carrier. ${ }^{[5-7]}$

The oral bioavailability of ACY is around $10-30 \%$ due to its poor water solubility and its incomplete absorption in GIT. ${ }^{[3]}$ An increase in aqueous solubility and absorption was observed in SDs of ACY. The enhanced drug release from the SDs may be related to the hydrophilic nature of carrier. It was also postulated that PEG20000 would have gelatinized in dissolution medium and might have held the drug particles in intimate contact with water (owing to its high water retention potential). This gelatinized SDs might have been constantly crushed by attrition during stirring, and the drug might have diffused into the dissolution medium through the polymeric diffusion layer. ${ }^{[4,6,7]}$ The " $n$ " values of the SDs in Korsmeyer-Peppas model was found to be between 0.257 and 0.388 and it indicated a non-Fickian release behavior of ACY from SDs. ${ }^{[8,15]}$

Sharp and intense characteristic peaks [Figure 2a] in X-ray diffraction spectra of ACY revealed its crystalline nature. ${ }^{[7]}$ Two distinct peaks in PEG20000 spectra proved its amorphous nature. Numerous sharp characteristic peaks of ACY were found to be absent in spectra of all SDs. Further, the two prominent peaks in sample spectra were found with a broad base and reduced peak intensity, sharpness as compared to the corresponding peaks in ACY spectra. These findings clearly suggest that the crystallinity of ACY was reduced/lost in SDs, and alteration of structural morphology might have taken place in the ACY molecule during the dispersions process. ${ }^{[16,17]}$ These postulations were also further supported by the findings of PMI images [Figure 3] which confirmed the decrease in melting point of ACY from $255^{\circ} \mathrm{C}$ to $170^{\circ} \mathrm{C} \cdot{ }^{[18]}$

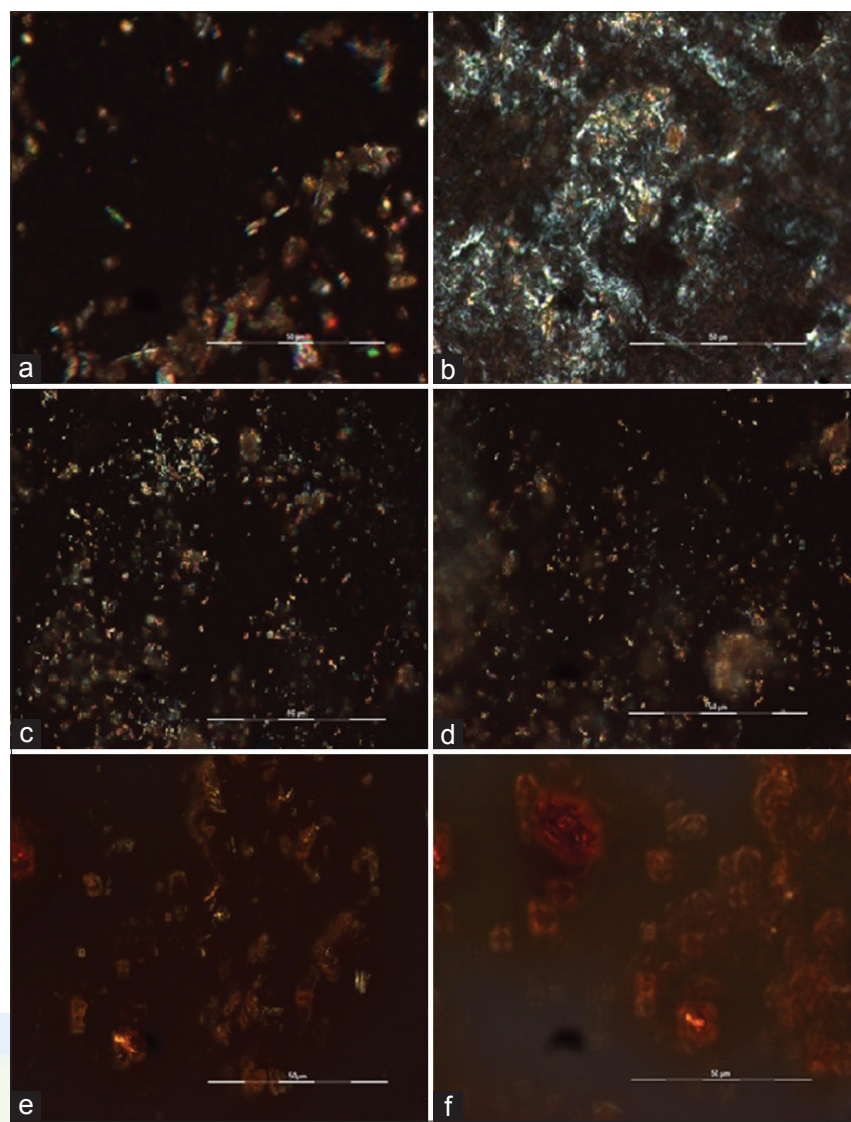

Figure 4: Polarizing microscopic images of (a) acyclovir, (b) polyethylene glycol 20000 melting at $56^{\circ} \mathrm{C}$, (c and d) APEG20 1:20 melting at $150^{\circ} \mathrm{C}$ and $160^{\circ} \mathrm{C}$, (e) acyclovir decomposition at $257^{\circ} \mathrm{C}$ and (f) acyclovir cooled to $25^{\circ} \mathrm{C}$

Table 4: Nuclear magnetic resonance data of acyclovir and optimized solid dispersions

\begin{tabular}{lcccccc}
\hline Sample code & $\mathbf{N 1 - H}$ & $\mathbf{H 8}$ & $\mathbf{N H}_{2}$ & $\mathbf{H 1 0}$ & $\mathbf{O H}$ & $\mathbf{H 1 1 / H 1 2}$ \\
\hline ACY & 10.687 & 7.820 & 6.509 & 5.465 & 4.703 & 3.517 \\
APEG20 120 & 8.656 & 7.202 & 6.849 & - & 3.501 & 2.513 \\
\hline
\end{tabular}

ACY: Acyclovir

Table 5: In situ permeation data of acyclovir
and optimized solid dispersions
\begin{tabular}{lcc}
\hline Code & $\boldsymbol{P}_{\text {eff }}(\mathbf{c m} / \mathbf{s}) \times 10^{-4}$ & ER \\
\hline ACY & 0.2860 & - \\
APEG20 120 & 1.0888 & 3.807 \\
\hline
\end{tabular}

$P_{\text {efff }}$ Effective permeability coefficient in $1 \mathrm{~h}$, ER: Enhancement ratio, ACY: Acyclovir

A sharp endothermic peak at $256^{\circ} \mathrm{C}$ in ACY thermogram [Figure 2b] revealed its high crystallinity. ${ }^{[3]} \mathrm{A}$ broad endothermic peak at $54.32^{\circ} \mathrm{C}$ in carrier thermogram suggested its amorphous nature. ${ }^{[17]}$ The endothermic peaks corresponding to ACY were found to be absent in thermograms of SDs. This thermal behavior may be related to the dissolution of ACY in the molten carrier the formation of solid solution of drug within the carrier. ${ }^{[4-7,16,19]}$

The characteristic peaks of ACY were absent in IR spectra [Figure 2c] of SDs, in comparison with pure ACY. This 


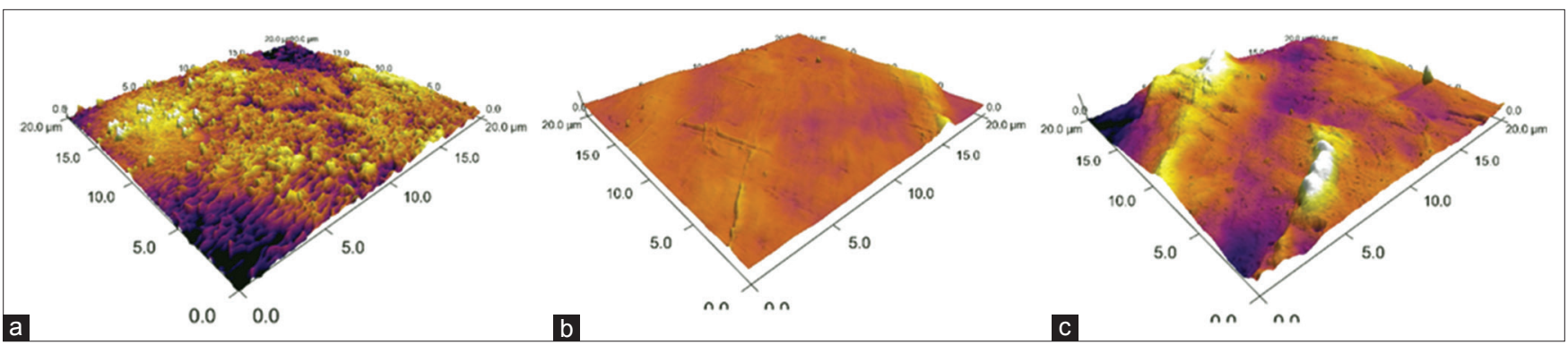

Figure 5: Atomic force microscopy images of (a) acyclovir; (b) polyethylene glycol 20000 and (c) APEG20 120

may be due to the dissolution of ACY in molten carrier and formation of a solid solution. ${ }^{[4-7,15,16,19]}$

The agglomerated image [Figure 4] of optimized SDs in SEM analysis and their reduced particle size indicated that the structural morphology of ACY had been altered during the formulation of SDs. ${ }^{[4-7]}$ Further, the parameters such as roughness data, three-dimensional surface profile and the absence of peaks in AFM images [Figure 5] clearly confirms the changes that had occurred in SDs at the molecular level in comparison to pure ACY. The mapping of ACY SDs at the molecular level by AFM analysis supported the above-suggested findings by SEM analysis. ${ }^{[11,12]}$

The enhanced $P_{\text {eff }}$ values, enhancement ratio from perfusion study and amount of drug diffused from permeability study of the optimized SDs proved their absorption potential in comparison with pure ACY. These findings could be correlated to the effect of carrier on increasing the aqueous solubility of ACY. ${ }^{[13,14]}$

It has been reported that more than one mechanism contributes to the solubility enhancement of drugs in SDs. Solid state characterization findings also suggested that the drug might have molecularly dispersed in the carrier structure, resulting in the formation of a solid solution and contributed for enhancement of drug's dissolution rate. This inference can be further strengthened by the DSC findings, which exhibited a single $\mathrm{Tg}$ for the SDs. The changes in surface morphology of the drug would have also led to the amorphization of the drug in carrier and this would have led to the higher dissolution, as it was evident from the SEM and AFM images of the optimized SDs. Hence, the decreased mobility of the components of SDs would have enhanced the physical stability of the prepared SDs. This interpretation was based on the results of NMR analysis and DSC findings. Based on the findings, the formulated SDs may be classified under Class A-C and Class M-C systems..$^{[1,2]}$ It was also suggested that semi-crystalline carriers such as PEG favors the formation of mixed systems, and the study findings were in correlation with the published reports. ${ }^{[1,2,9,10,20]}$

\section{CONCLUSION}

The comparison of the dissolution rate of ACY with its corresponding SDs indicated a high release profile of the SDs. Solid state characterization studies provided complete mechanistic insights into the possible mechanism of enhanced drug release from SDs. The single-pass intestinal perfusion and intestinal permeation studies in rats confirmed that optimized SDs displayed a good absorption potential. It can be concluded that the results demonstrated SDs of ACY's aqueous solubility, dissolution and its subsequent absorption could be significantly improved by dispersing in a carrier like PEG20000.

\section{Acknowledgments}

The authors acknowledge the support of CCM Duopharma Sdn Bhd and Dynapharma Sdn Bhd in providing samples of acyclovir.

\section{Financial support and sponsorship}

Nil.

\section{Conflicts of interest}

There are no conflicts of interest.

\section{REFERENCES}

1. Vo CL, Park C, Lee BJ. Current trends and future perspectives of solid dispersions containing poorly water-soluble drugs. Eur J Pharm Biopharm 2013;85(3 Pt B):799-813.

2. Meng F, Gala U, Chauhan H. Classification of solid dispersions: Correlation to (i) stability and solubility (ii) preparation and characterization techniques. Drug Dev Ind Pharm 2015;41:1401-15.

3. Attia IA, El-Gizawy SA, Fouda MA, Donia AM. Influence of a niosomal formulation on the oral bioavailability of acyclovir in rabbits. AAPS PharmSciTech 2007;8:E106.

4. Khattab IS, Nada A, Zaghloul AA. Physicochemical characterization of gliclazide-macrogol solid dispersion and tablets based on optimized dispersion. Drug Dev Ind Pharm 2010;36:893-902.

5. Arias MJ, Moyano JR, Ginés JM. Study by DSC and HSM of the oxazepam-PEG 6000 and oxazepam-D-Mannitol systems: Application to the preparation of solid dispersions. Thermochim Acta 1998;321:33-41.

6. Biswal S, Sahoo J, Murthy PN, Giradkar RP, Avari JG. Enhancement of dissolution rate of gliclazide using solid dispersions with polyethylene glycol 6000 . AAPS PharmSciTech 2008;9:563-70.

7. Ahuja N, Katare OP, Singh B. Studies on dissolution enhancement and mathematical modeling of drug release of a poorly water-soluble drug using water-soluble carriers. Eur J Pharm Biopharm 2007;65:26-38.

8. Zhang Y, Huo M, Zhou J, Zou A, Li W, Yao C, et al. DDSolver: An add-in program for modeling and comparison of drug dissolution profiles. AAPS J 2010;12:263-71. 
9. Janssens S, Van den Mooter G. Review: Physical chemistry of solid dispersions. J Pharm Pharmacol 2009;61:1571-86.

10. Laitinen R, Suihko E, Toukola K, Björkqvist M, Riikonen J, Lehto VP, et al. Intraorally fast-dissolving particles of a poorly soluble drug: Preparation and in vitro characterization. Eur J Pharm Biopharm 2009;71:271-81.

11. Lauer ME, Siam M, Tardio J, Page S, Kindt JH, Grassmann O. Rapid assessment of homogeneity and stability of amorphous solid dispersions by atomic force microscopy - From bench to batch. Pharm Res 2013;30:2010-22.

12. Qian F, Huang J, Hussain MA. Drug-polymer solubility and miscibility: Stability consideration and practical challenges in amorphous solid dispersion development. J Pharm Sci 2010;99:2941-7.

13. Janga $K Y$, Jukanti $R$, Velpula A, Sunkavalli $S$, Bandari $S$, Kandadi $\mathrm{P}$, et al. Bioavailability enhancement of zaleplon via proliposomes: Role of surface charge. Eur J Pharm Biopharm 2012;80:347-57.

14. Zakeri-Milani $\mathrm{P}$, Valizadeh $\mathrm{H}$, Tajerzadeh $\mathrm{H}$, Azarmi $\mathrm{Y}$, Islambolchilar Z, Barzegar S, et al. Predicting human intestinal permeability using single-pass intestinal perfusion in rat.
J Pharm Pharm Sci 2007;10:368-79.

15. Barzegar-Jalali $M$, Adibkia $K$, Valizadeh $H$, Shadbad MR, Nokhodchi A, Omidi Y, et al. Kinetic analysis of drug release from nanoparticles. J Pharm Pharm Sci 2008;11:167-77.

16. Barmpalexis $P$, Kachrimanis K, Georgarakis E. Physicochemical characterization of nimodipine-polyethylene glycol solid dispersion systems. Drug Dev Ind Pharm 2014;40:886-95.

17. Newa M, Bhandari KH, Kim JA, Yoo BK, Choi HG, Yong CS, et al. Preparation and evaluation of fast dissolving ibuprofen-polyethylene glycol 6000 solid dispersions. Drug Deliv 2008;15:355-64.

18. Arias MJ, Gines JM, Moyano JR, Perez-Martinez JI, Rabasco AM. Influence of the preparation method of solid dispersions on their dissolution rate: Study of triamterene-D-mannitol system. Int J Pharm 1995;123:25-31.

19. Biswal S, Sahoo J, Murthy PN. Characterisation of gliclazide-PEG 8000 solid dispersions. Trop J Pharm Res 2009;8:417-24.

20. Vasconcelos T, Sarmento B, Costa P. Solid dispersions as strategy to improve oral bioavailability of poor water soluble drugs. Drug Discov Today 2007;12:1068-75. 